\title{
Coded Liquid Crystal Metasurface for Achromatic Imaging in the Broadband Wavelength Range
}

\author{
Qian Xu, ${ }^{1,2}$ Ti Sun ${ }^{1,2}$, and Chinhua Wang, 2,* \\ ${ }^{1}$ School of Optoelectronics Science and Engineering and Collaborative Innovation \\ Center of Suzhou Nano Science and Technology, Soochow University, Suzhou \\ 215006, China \\ ${ }^{2}$ Key Lab of Advanced Optical Manufacturing Technologies of Jiangsu Province and \\ Key Lab of Modern Optical Technologies of Education Ministry of China, Soochow \\ University, Suzhou 215006, China \\ *chinhua.wang@suda.edu.cn
}

\section{Section 1. Discuss on the Effect of Phase Coding Parameter}

The effect of the coding parameter $\alpha$ on the performance of the broadband achromatic imaging of a C-LCL includes essentially the consistency of MTFs and the signal-tonoise ratio of the system.

The consistency of MTFs of a C-LCL system (focal length $100 \mathrm{~mm}$, diameter $4 \mathrm{~mm}$, designed wavelength $633 \mathrm{~nm}$ ) at different wavelengths can be evaluated by the mean 
squared error (MSE) between the $\mathrm{MTF}_{\Delta \lambda}$ of the C-LCL at wavelength deviation $\Delta \lambda$ and the $\mathrm{MTF}_{0}$ of the C-LCL at designed wavelength:

$$
M S E=\sqrt{\frac{\sum_{i=1}^{N}\left(M T F_{\Delta \lambda}-M T F_{0}\right)^{2}}{N}}
$$

where $N$ is the number of sampling points at different spatial frequencies. According to equation (S1), Figure S1a shows the MSE of the C-LCL at wavelength deviation $\Delta \lambda=67 \mathrm{~nm}$ for coding parameters ranging from $\alpha=0$ to $\alpha=100 \pi$. With the increase of coding parameter $\alpha$, MSE decreases, which means the consistency of MTFs within the fixed bandwidth increases, i.e., operation bandwidth of the C-LCL increases. Figure S1b shows the MTFs with different coding parameters. It is seen that with the increase of the coding parameter, the MTF decreases, which means decreased signal to noise ratio of the system. Therefore, a balance between the bandwidth and the signal to noise ratio of the system should be considered when a coding parameter is employed.
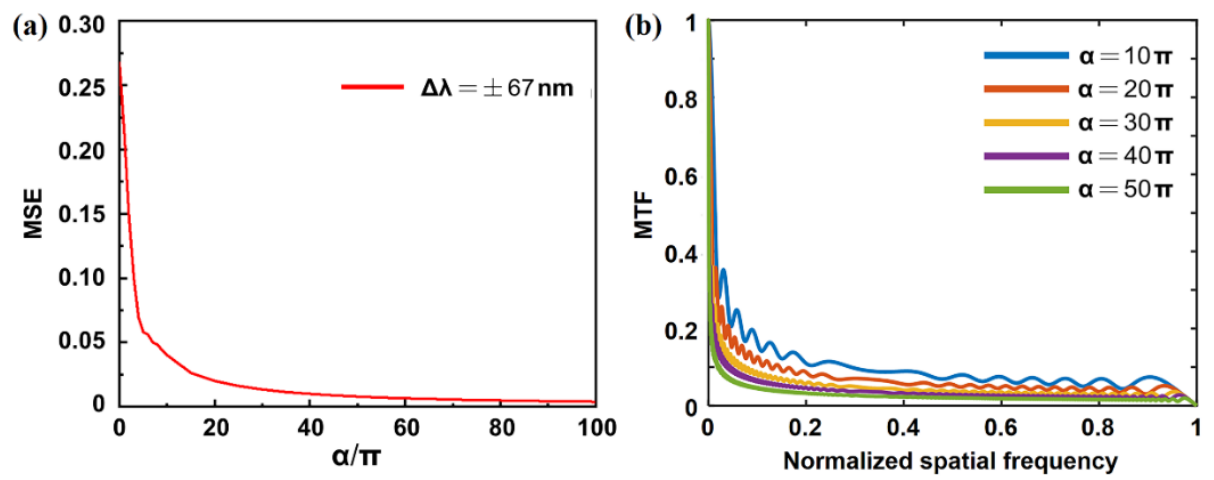

Figure S1. (a) MSE of the C-LCL at wavelength deviation $\Delta \lambda=67 \mathrm{~nm}$ for coding parameters ranging from $\alpha=0$ to $\alpha=100 \pi$. (b) MTF of the C-LCL with different coding parameters. 


\section{Section 2. Fabrication Process of the LC Lenses}

LC lenses were fabricated using photopatterning technique. Materials we used in the experiment are nematic liquid crystal (E7, SLiC) and azo dye methyl red (MR, sigmaaldrich). They were mixed with a ratio of $99: 1$. When illuminated by linearly polarized light, the MR molecules will transform from trans to cis, and end up in perpendicular to the incidence polarization direction [1]. The alignment of the MR molecules, subsequently, determines the alignment of the LC molecules (i.e., orientation angle $\beta$ ), from which the phase profile of the LC metasurface can be generated. The transmission spectrum of LC/MR mixture are measured and shown in Figure S2a, which is similar to that in ref. [2]. It can be seen that it has strong absorption in green light region and high transmission in the red light region. Therefore, a 532nm laser was chosen as the exposure light and working wavelength of imaging was chosen in the red light region. Then the mixture was injected into a LC cell $(3 \mu \mathrm{m})$ while heated at around $60^{\circ} \mathrm{C}$. Figure $\mathrm{S} 2 \mathrm{~b}$ is the photopatterning setup in which a target phase profile (i.e., grey scale patterns as shown in Figure 1a or b) were input to a spatial light modulator (SLM, model PLUTO-VIS, Holoeye, 1920x1080, pixel size $8 \mu \mathrm{m}$ ) through a computer, and then were converted to electric field patterns, i.e., orientation patterns of the LC molecules, with the help of a polarizer (P) and a quarter waveplate $(\lambda / 4)$.

The detailed relationship between the grey scale and the orientation angle of the LC molecules in our system can be established and calibrated by using a series of uniform gray scale patterns on the SLM and recording the corresponding polarization angle of 
the output linear polarization ((THORLABS, PAN5710VIS). Figure S2c gives the measured relationship between angle of the output linearly polarized light and the greylevels, which exhibits an excellent linear behavior. Based on Figure S2c, designed LC lenses can be fabricated by loading target phase profile with the corresponding calibrations of the system. Figure S2d-e are, respectively, the grayscale pattern of the U-LCL and C-LCL loaded on the spatial light modulator, in which an increment angle of $15^{\circ}$ in the orientation was used. In order to obtain a balanced pixel resolution and the aperture size of the lens, the position of lens L 2 in the system can be adjusted, in which a magnification factor of " 0.5 " was used to generate an aperture size of $4 \mathrm{~mm}$ in our case. During the exposure, the $\mathrm{LC}$ cell filled with mixture was heated to $67^{\circ} \mathrm{C}$ to ensure that the MR molecules can diffuse and be fixed on substrates [1]. The exposure time was about 10 minutes with an intensity of $\sim 42 \mathrm{mw} / \mathrm{cm}^{2}$ exposure power.
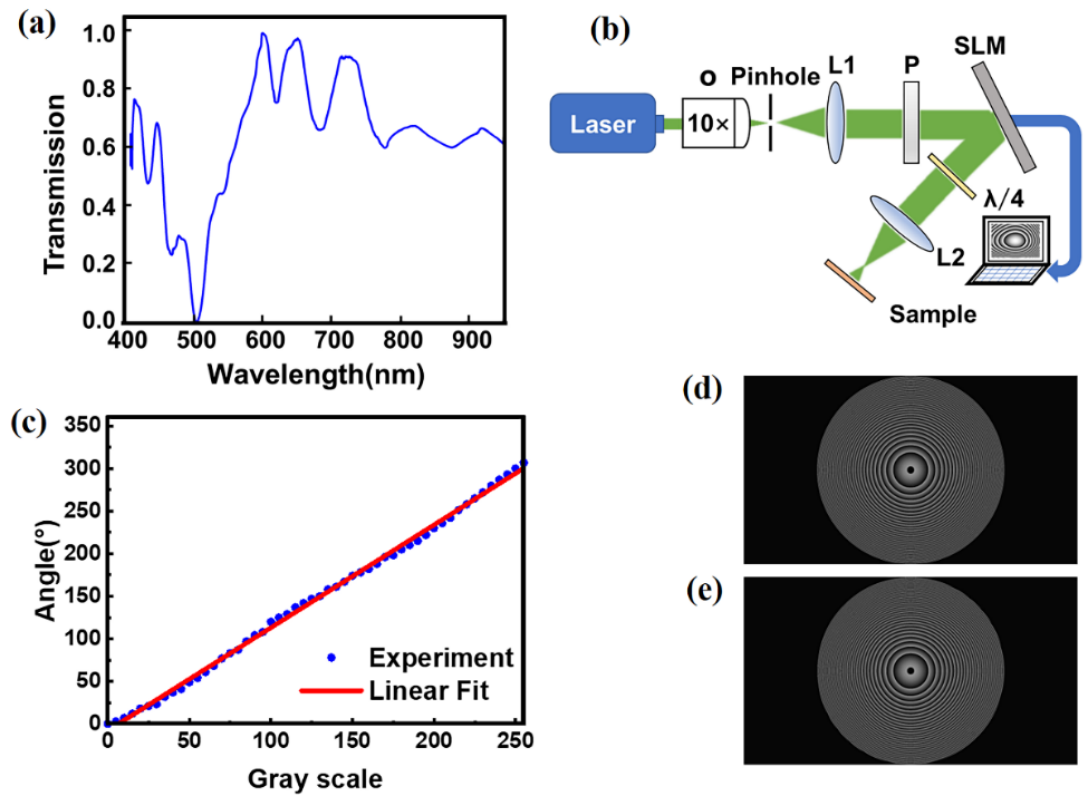

(d)

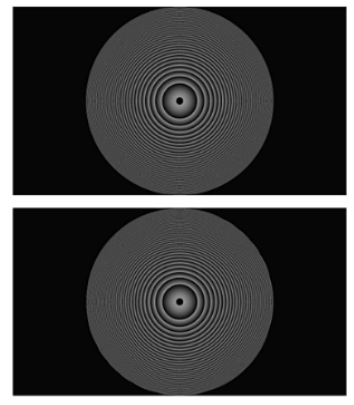

Figure S2. Normalized absorptance and transmittance of LC/MR mixture. (b) Optical setup for patterning exposure. (c) Angle of linearly polarized light versus grey-levels. 
(d) Grayscale pattern of U-LCL loaded on the spatial light modulator. (e) Grayscale pattern of C-LCL loaded on the spatial light modulator.

\section{Section 3. Optical Setup for LC Lens Imaging}

To demonstrate the capability of broadband achromatic and wide FOV imaging with the proposed and fabricated C-LCL, an optical imaging system was set up as shown in Figure S3. By adjusting the reflecting mirror, the source switch between monochromatic light $(633 \mathrm{~nm})$ and broadband Tungsten lamp can be made. Different optical filters with the required wavelength bands were used for the broadband imaging with the Tungsten lamp (THORLABS, FESH0700, FEL0600, FL632,8-10). Wideband circular polarizers of LCP and RCP (Edmund, CP42HE, CP42HER) were employed to convert the source to circularly polarized light and filter the non-diffractive background ghost image. A collimator (diameter $55 \mathrm{~mm}$ and focal length $550 \mathrm{~mm}$ ) was used to generate the collimated light. Due to the requirement of the P-B phase, an optimized voltage $(2.5 \mathrm{~V}$ in our case) was applied to the LC cell to optimize the diffraction efficiency. At the focal plane, a CCD with pixel size 4.54 $\mu$ m (AVT Prosilica GX2750C) was placed on the same rotation stage with the LC lenses. For the designed U-LCL $(f / 25)$ under $633 \mathrm{~nm}$ wavelength, the airy radius is expected to be $19.3 \mu \mathrm{m}$ calculated by

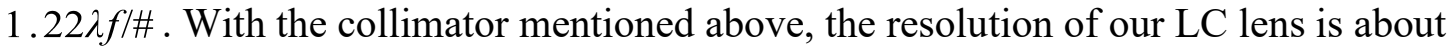
$9.42 \mathrm{lp} / \mathrm{mm}$ from res $=\frac{D_{\mathrm{PBL}}}{1.22 \lambda f_{\mathrm{PBL}}} / \frac{f_{\text {collimator }}}{f_{\mathrm{PBL}}}$, where $D_{\mathrm{PBL}}$ is the diameter of LC lens, $f_{\mathrm{PBL}}$ is the focal length of LC lens, $\lambda$ is the designed wavelength, and $f_{\text {collimator }}$ is the focal length of the collimator. 


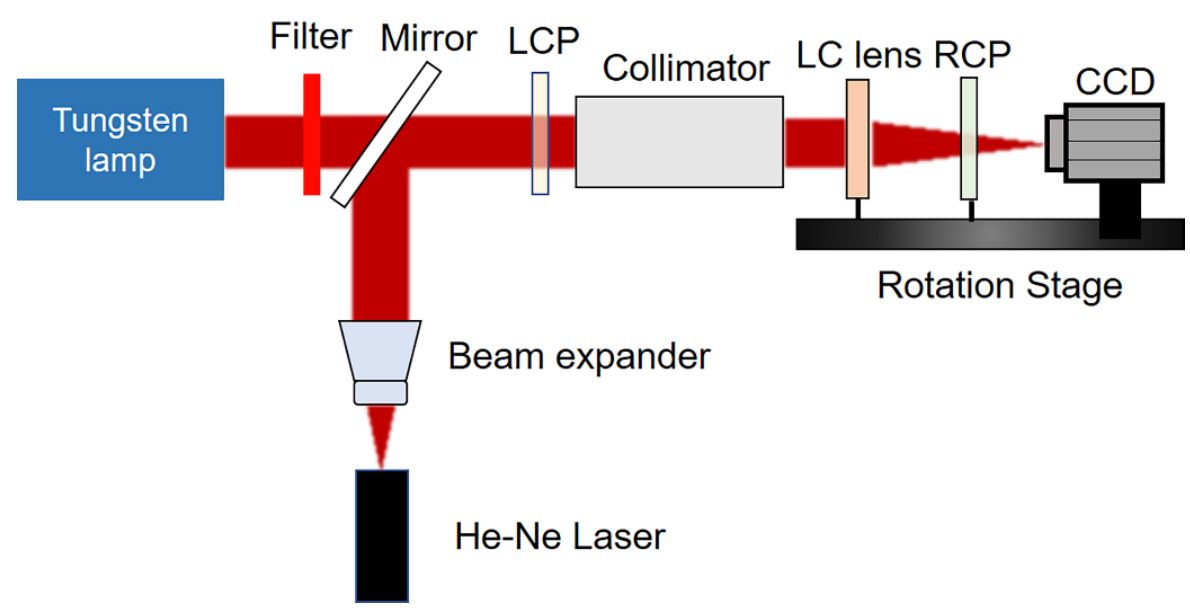

Figure S3. Optical setup for LC lens imaging.

\section{Section 4. Imaging at Different Wavelengths in the Achromatic Wavelength Range}

Imaging at different wavelengths within the achromatic wavelength range from 566 to $700 \mathrm{~nm}$ with U-LCL and C-LCL, respectively, are measured and compared in Figure S4-S6.

With no doubt, the PSFs (Figure S4a) and imaging (Figure S4b) of U-LCL are diffused when working wavelength deviates from the designed wavelength $633 \mathrm{~nm}$. In contrast, the PSFs (Figure S5a) and imaging (Figure S5b) of C-LCL remain consistent (although all the images are blurred) when working wavelength changes within the achromatic range. These blurred intermediate images can be restored using filtering process. The intermediate images (Figure S5b) were deconvolved with the PSF of 633 $\mathrm{nm}$ (Figure S5a), the restored images were given in Figure S6a, and it is seen that all the restored images at any wavelength within the working bandwidth are clear. In comparison, the same deconvolution process was performed for the images obtained with U-LCL, and the restored results were given in Figure S6b. It is seen that no clear 
images can be obtained after the filtering process because of fact that zeros in the MTFs of the U-LCL appear once bandwidth is beyond $10 \mathrm{~nm}$, which means that spatial frequency information of the image is lost and cannot be recovered by restoration. It is noted that the image quality after restoration at designed wavelength $633 \mathrm{~nm}$ in the case of U-LCL becomes even worse compared to the original image (Figure S4b) because of the noises introduced during the filtering process.
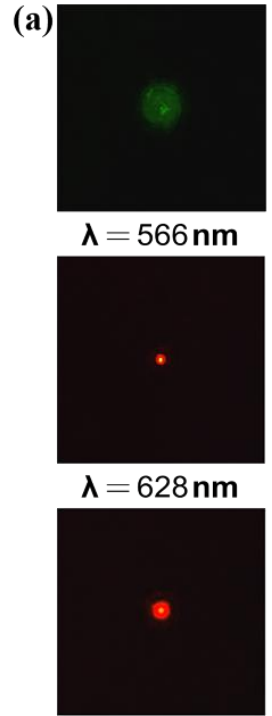

$\lambda=666 \mathbf{n m}$

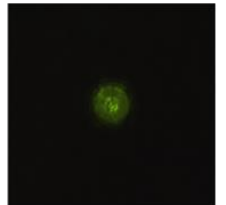

$\lambda=576 \mathrm{~nm}$

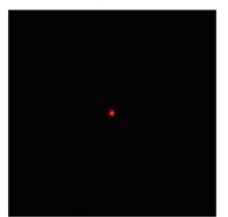

$\lambda=633 \mathbf{n m}$

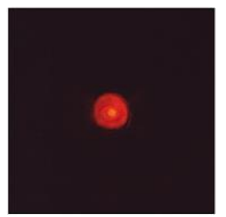

$\boldsymbol{\lambda}=690 \mathrm{~nm}$

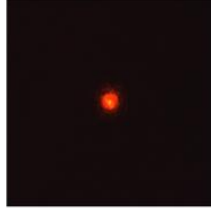

$\lambda=600 \mathrm{~nm}$

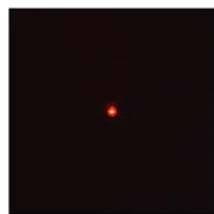

$\lambda=638 \mathrm{~nm}$

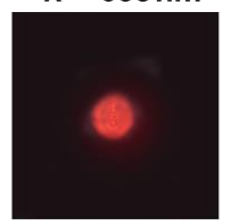

$\lambda=700 \mathrm{~nm}$

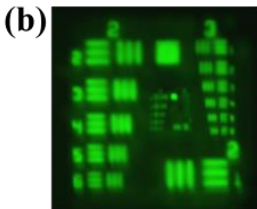

$\lambda=566 \mathrm{~nm}$

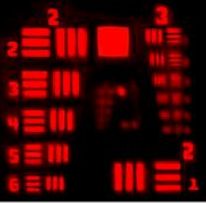

$\lambda=628 \mathrm{~nm}$

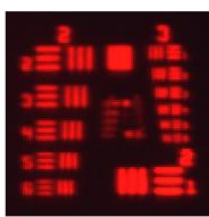

$\lambda=666 \mathrm{~nm}$

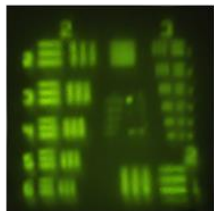

$\lambda=576 \mathrm{~nm}$

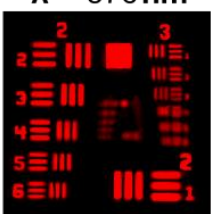

$\lambda=633 \mathrm{~nm}$

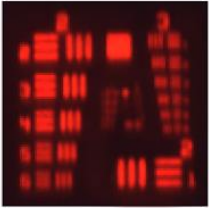

$\boldsymbol{\lambda}=690 \mathrm{~nm}$

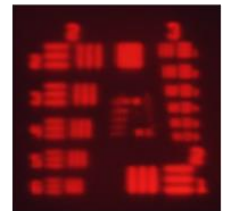

$\lambda=600 \mathrm{~nm}$

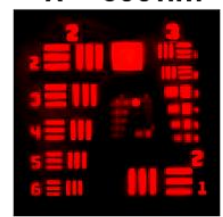

$\lambda=638 \mathrm{~nm}$

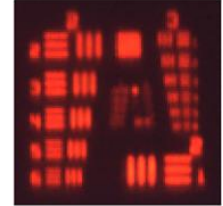

$\boldsymbol{\lambda}=700 \mathrm{~nm}$

Figure S4. (a) PSFs and (b) imaging at different wavelengths in $566 \mathrm{~nm}-700 \mathrm{~nm}$ range with U-LCL. 
(a)

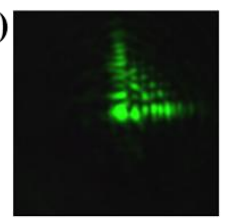

$\lambda=566 \mathrm{~nm}$

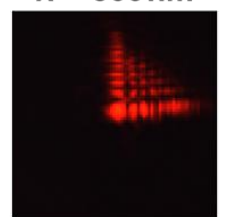

$\lambda=628 \mathrm{~nm}$

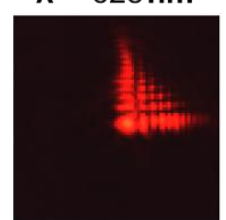

$\lambda=666 \mathrm{~nm}$

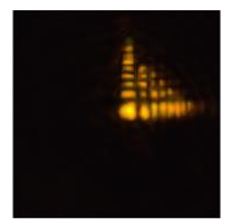

$\boldsymbol{\lambda}=576 \mathrm{~nm}$

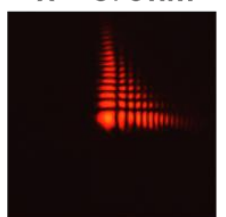

$\lambda=633 \mathrm{~nm}$

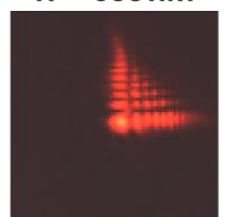

$\boldsymbol{\lambda}=690 \mathrm{~nm}$

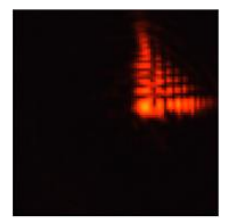

$\lambda=600 \mathrm{~nm}$

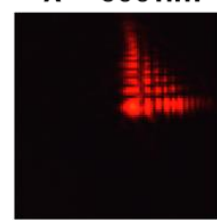

$\lambda=638 \mathrm{~nm}$

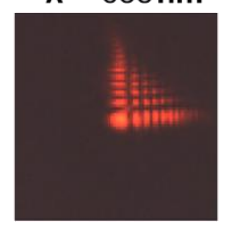

$\boldsymbol{\lambda}=700 \mathrm{~nm}$

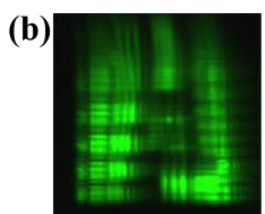

$\lambda=566 \mathrm{~nm}$

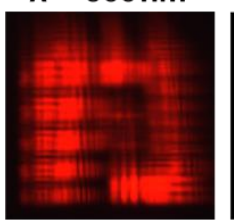

$\lambda=628 \mathrm{~nm}$

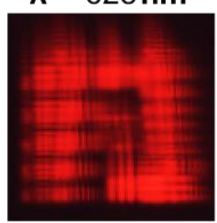

$\boldsymbol{\lambda}=666 \mathrm{~nm}$

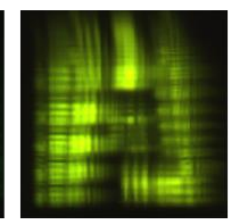

$\lambda=576 \mathrm{~nm}$

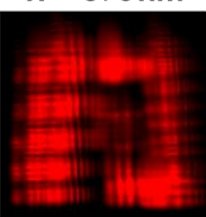

$\lambda=633 \mathrm{~nm}$

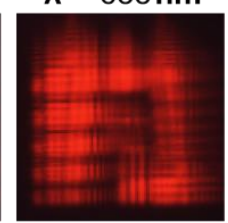

$\boldsymbol{\lambda}=690 \mathbf{n m}$

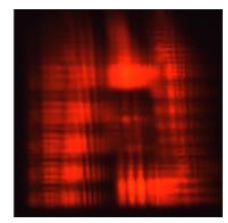

$\lambda=600 \mathrm{~nm}$

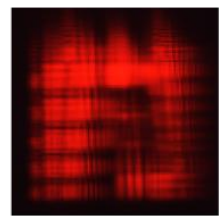

$\boldsymbol{\lambda}=638 \mathrm{~nm}$

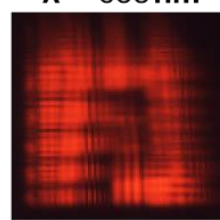

$\boldsymbol{\lambda}=700 \mathrm{~nm}$

Figure S5. (a) PSFs and (b) imaging at different wavelengths in $566 \mathrm{~nm}-700 \mathrm{~nm}$ range with C-LCL.

(a)
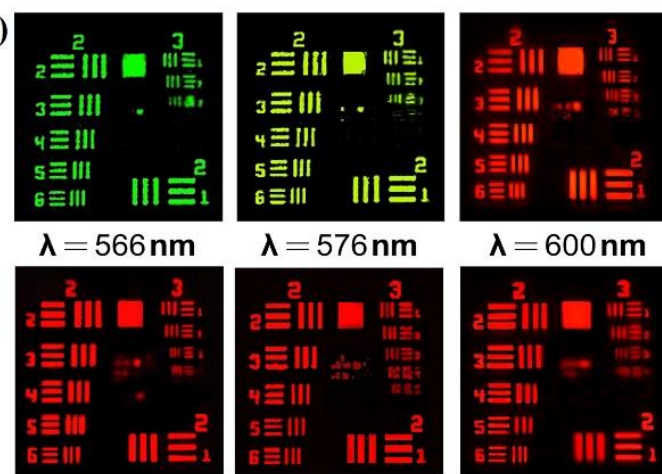

$\lambda=628 \mathrm{~nm}$

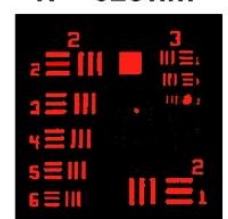

$\boldsymbol{\lambda}=666 \mathrm{~nm}$

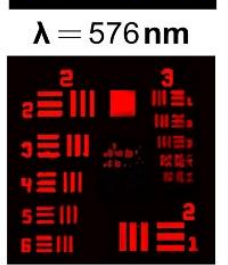

$\lambda=633 \mathrm{~nm}$

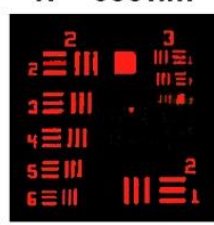

$\boldsymbol{\lambda}=690 \mathrm{~nm}$
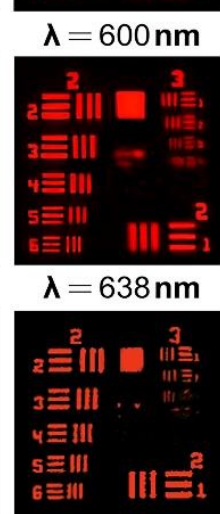

$\boldsymbol{\lambda}=700 \mathrm{~nm}$
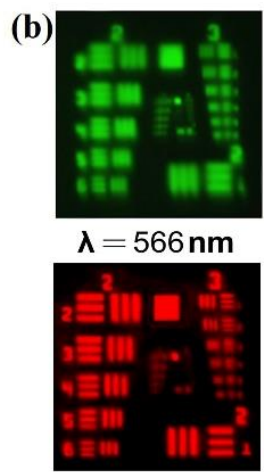

$\lambda=628 \mathrm{~nm}$

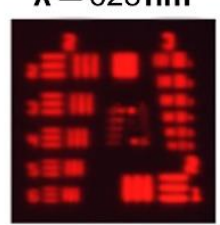

$\lambda=666 \mathrm{~nm}$

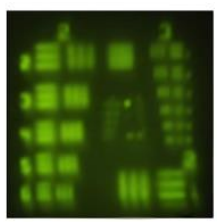

$\boldsymbol{\lambda}=576 \mathrm{~nm}$

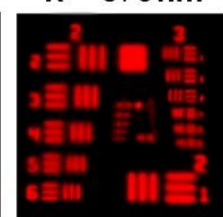

$\lambda=633 \mathbf{n m}$

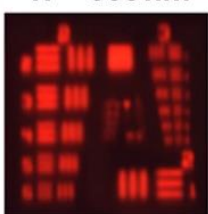

$\boldsymbol{\lambda}=690 \mathrm{~nm}$

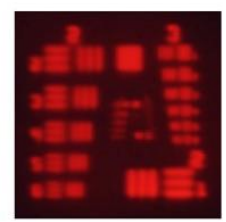

$\lambda=600 \mathrm{~nm}$

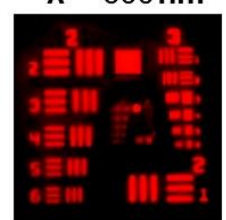

$\lambda=638 \mathrm{~nm}$

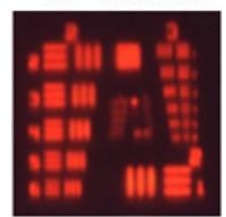

$\boldsymbol{\lambda}=700 \mathrm{~nm}$

Figure S6. Restored imaging at different wavelengths in $566 \mathrm{~nm}-700 \mathrm{~nm}$ range with (a)

C-LCL; and (b) U-LCL. 


\section{Section 5. Diffraction Efficiency Versus Wavelengths}

Under $100 \mathrm{~nm}$ broadband illumination, the optical setup for measuring diffraction efficiency is shown in Figure S7. When illuminated by monochromatic wavelength, tungsten lamp and filter were replaced by a continuously tunable laser (Fianium, LLTFCONTRAST VIS-2). Wideband circular polarizers of LCP and RCP (Edmund, CP42HE, CP42HER) were employed to convert the source to circularly polarized light and filter the non-diffractive background ghost image.

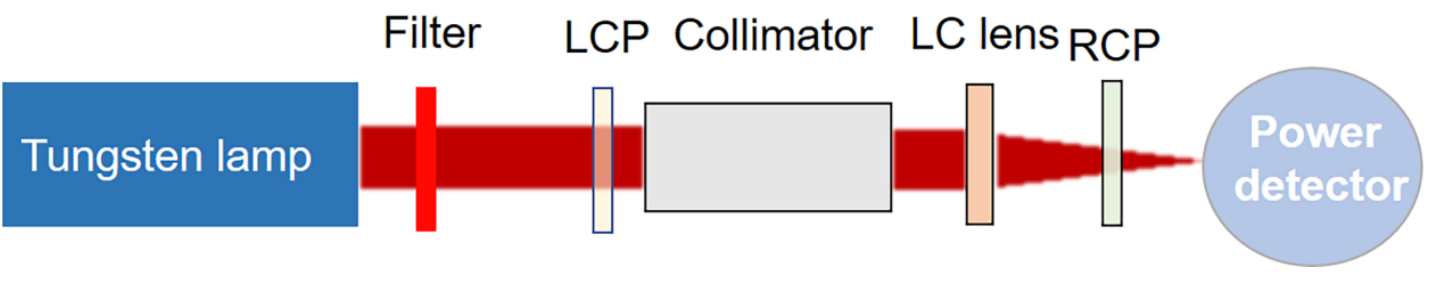

Figure S7. Optical setup for measuring diffraction efficiency.

When a fixed voltage of $2.5 \mathrm{~V}$ was applied, solid lines (red and black) in Figure S8 show the diffraction efficiency versus wavelength with U-LCL and C-LCL, respectively. It is seen that the maximum efficiency for U-LCL and C-LCL are 85\% and $84 \%$, respectively, at the designed wavelength $633 \mathrm{~nm}$, which is about $6 \%$ higher than that with a broadband illumination. The efficiency decreases rapidly as the wavelength decreases, which attributes to the absorption of MR for green light. If the diffraction efficiency at different wavelengths were measured with an optimized voltage at different wavelengths to make the phase retardation meet the half-wave retardation condition, maximized diffraction efficiencies at different wavelengths can be obtained, as shown with the dashed line in Figure S8. It is seen from the dashed lines that all the efficiencies are greater than $80 \%$, which is similar to those reported results 
when actually 12-step discrete P-B phase and imperfection exposure in the fabrication were considered.

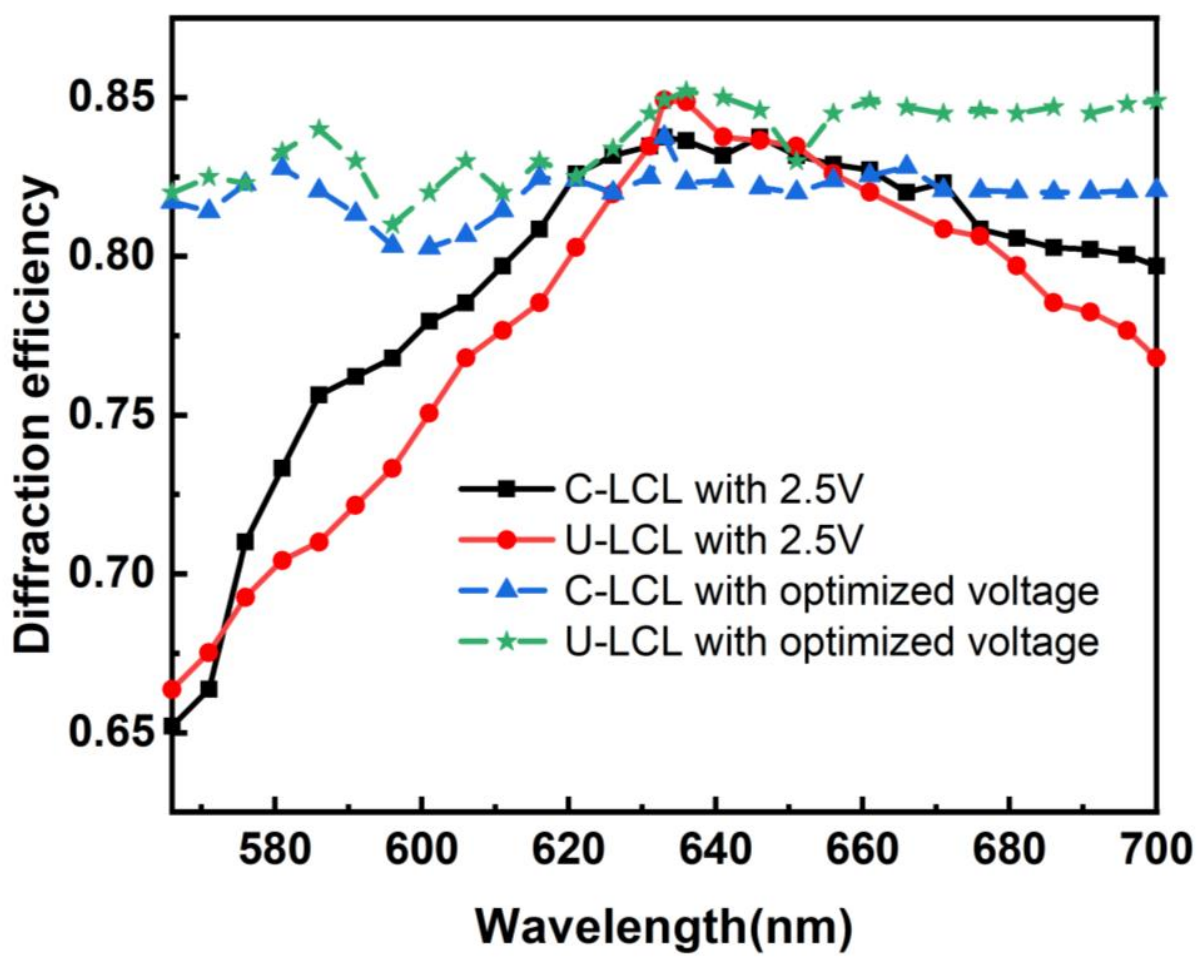

Figure S8. Diffraction efficiency versus wavelengths with fixed voltage of $2.5 \mathrm{~V}$ or optimized voltages at different wavelengths with U-LCL and C-LCL, respectively.

[1]. L.-C. Lin, H.-C. Jau, T.-H. Lin, and A. Y. G. Fuh, "Highly efficient and polarization-independent Fresnel lens based on dye-doped liquid crystal," Optics Express, 15(6), 2900-2906 (2007).

[2]. S. Li, Y. Liu, Y. Li, S. Liu, S. Chen, and Y. Su, "Fast-response PancharatnamBerry phase optical elements based on polymer-stabilized liquid crystal," Optics Express, 27(16), 22522-22531 (2019). 